\begin{tabular}{|l|l|l|l|l|}
\hline \multirow{2}{*}{ aptara } & APA & apa486 & Dispatch: August 24, 2007 & CE: AM \\
\cline { 2 - 5 } & Journal & MSP No. & No. of pages: 5 & PE: Ruth \\
\hline
\end{tabular}

\title{
Cry features in siblings of SIDS
}

\author{
Michael P Robb (michael.robb@canterbury.ac.nz)ํ, David H Crowell², Peter Dunn-Rankin³ , Cynthia Tinsley ${ }^{4}$ \\ 1.Department of Communication Disorders, University of Canterbury, Christchurch, New Zealand \\ 2.Department of Pediatrics, University of Hawaii at Manoa and Kapiolani Medical Center for Women and Children, Honolulu, Hawaii, USA \\ 3.College of Education, University of Hawaii at Manoa, Honolulu, Hawaii, USA \\ 4.Department of Pediatrics, Loma Linda University Children's Hospital, Loma Linda, California, USA
}

\section{Keywords}

Acoustic analysis, Cry, Siblings, SIDS

\section{Correspondence}

Michael P Robb, Department of Communication

Disorders, University of Canterbury, Private Bag

4800, Christchurch, New Zealand.

Tel: $011-643-364-2987$ |

Fax: 011-643-364-2760

Email: michael.robb@canterbury.ac.nz

Received

18 June 2007; revised 19 July 2007; accepted 25 July 2007.

DOI:10.1111/j.1651-2227.2007.00486.x

\begin{abstract}
Aim: To examine the acoustic features of crying demonstrated by infants whose older sibling died of sudden infant death syndrome (SIDS) and compare these features to a nonrisk group of infants. Methods: Pain-induced crying episodes were collected from a group of healthy term (HT) infants and siblings of SIDS infants. One complete crying episode was obtained from each infant and analyzed acoustically with regard to durational and spectral features.

Results: The cries of SIDS siblings were found to be significantly higher in pitch and reflected hyperadductory vocal fold vibratory behaviour compared to the HT group. There were no group differences with regard to durational features of crying.

Conclusions: The laryngeal behaviour of infant crying, as inferred via acoustic analyses, differs between HT infants and siblings of SIDS infants. Accordingly, acoustic features of infant crying may serve as an additional diagnostic marker in the identification of children who may be at risk for SIDS.
\end{abstract}

\section{INTRODUCTION}

Identifying the precise cause of sudden infant death syndrome (SIDS) remains elusive. A recent policy statement issued by the American Academy of Pediatrics (AAP) suggests that SIDS is caused by a maldevelopment or delay in maturation of the brainstem neural network that is responsible for arousal and affects physiological responses to lifethreatening challenges during sleep (1). The occurrence of SIDS in the United States has reduced by approximately $50 \%$ in the past 15 years, which has been attributed to the 'Back to Sleep' campaign advocated by the AAP. The major message proffered in this campaign is that infants should be placed for sleep in a wholly supine position.

Among industrialized countries, SIDS continues to be the number three cause of death after congenital malformations and disorders related to short gestation (2). The search for factors that may predispose an infant to SIDS has yielded a long list of environmental, anthropometric, familial and physiological correlates (3). One common line of investigation has been to examine various infant groups thought to be at a particularly high risk for SIDS. These groups include preterm infants, low birthweight infants, infants experiencing an idiopathic apparent life-threatening event (ALTE), as well as siblings of SIDS (4). Although the first two groups have been consistently identified as independent risk factors for SIDS (3), the link between SIDS and the latter two groups is less clear. For example, over 30 years ago Steinschneider (5) suggested that prolonged sleep apnea (an abnormality found in ALTE infants) was a contributing factor to SIDS. However, more recent research has not supported a direct relationship between the two events (6).

Rarest among these risk groups are siblings of SIDS babies, who by their very nature represent a small but unique categorization of infants. The risk of SIDS recurring in siblings is controversial. There is research to suggest that SIDS shows a strong sibship aggregation compared to the general population, indicating a genetic or biological predisposition (7). However, there is also research suggesting that the risk of a later sibling dying from SIDS is no higher than that of the general population (8). There is also a suggestion that SIDS siblings may be at a lower risk for SIDS compared to the general population. This scenario could be a consequence of parents more likely being acutely aware of the steps necessary to reduce risk, including the 'Back to Sleep' recommendation (9).

At present, there seems to be no unified agreement that siblings of SIDS infants demonstrate physiological or behavioural characteristics that differ from the general population of healthy term (HT) infants (3). The current study was designed to examine whether cry physiology served to differentiate SIDS siblings from HT infants. Acoustic analysis of crying behaviour provides a noninvasive means of correlating an infant's neurobehavioural integrity with stability of laryngeal coordination (10). More often than not, various acoustic features of the cries of unhealthy infants are clearly different from the cries of healthy infants (11).

On the basis of past physiological research sited above, one would predict that the crying behaviour of SIDS siblings would either (a) not differ from the general population of infants or (b) would parallel that of SIDS infants. At present there is a large database concerning the acoustic characteristics of crying in HT infants (12) but there are minimal studies evaluating the crying behaviour in SIDS. Stark and Nathanson (13) obtained cry samples from a 4-dayold infant who died of SIDS at 6 months of age. The infant's cries were found to be of shorter duration and having a higher pitch (e.g. fundamental frequency $=\mathrm{F}_{\mathrm{o}}$ ) than HT 
infants. In contrast, Colton and Steinschneider (14) evaluated the cries in one SIDS infant and found the cries to be of long duration, with a low $\mathrm{F}_{\mathrm{o}}$, and low vocal tract resonance (e.g. formant frequencies) compared to HT infants. Corwin et al. (15) examined 12 SIDS infants and obtained results that did not fully support either of the earlier two studies. Specifically, cry duration did not differ between SIDS and HT infants. However, the SIDS infants were found to have a high $\mathrm{F}_{\mathrm{o}}$, which confirmed the previous observation by Stark and Nathanson (but conflicts with Colton and Steinschneider). In addition, the SIDS infants examined by Corwin et al. produced cries with significantly higher formant frequencies compared to HT infants, which was opposite to the pattern reported by Colton and Steinschneider. Needless to say, a great deal remains to be resolved concerning the definitive crying behaviour of SIDS infants.

We found one comprehensive study evaluating the cry characteristics of SIDS siblings, the results of which suggest a subtle similarity to the cries of SIDS infants. Colton et al. (16) compared the cries of SIDS siblings to groups of HT and preterm infants during the first and fourth weeks of life. The researchers found SIDS siblings to differ from the comparison groups primarily during the first week of life. The SIDS siblings produced cries with significantly greater loudness in the high spectral regions $(4-8 \mathrm{kHz})$ compared to the other groups. In addition, the SIDS siblings produced cries with an average cry $F_{o}$ that was approximately 40 $\mathrm{Hz}$ lower (though statistically nonsignificant). These results were found to match the crying behaviour demonstrated in an SIDS infant; however, the spectral energy and $F_{o}$ was more extreme in the SIDS infant (14).

The current study examined the acoustic features of crying demonstrated by SIDS siblings compared to a group of HT infants. The study was motivated by a number of issues. First, there is uneven evidence to date that SIDS siblings present physiological or behavioural characteristics that are different from the general population of infants (4). Second, past research evaluating the crying behaviour of SIDS siblings are suggestive of abnormal laryngeal behaviour; however, this documentation is based on one study performed over 20 years ago. Third, past research evaluating the cries of SIDS infants have yielded conflicting results. Therefore, comparison of the cries of SIDS siblings with past results reported for SIDS infants may help to isolate the distinct features of crying in SIDS infants. The general research question posed in the present study was, "Do the cries of siblings of SIDS infants differ significantly from those of HT infants?"

\section{METHOD}

\section{Participants}

Data for the current study were collected as part of the Collaborative Home Infant Monitoring Evaluation (CHIME) Study, a program supported by the National Institute of Child Health and Human Development, National Institutes of Health. A primary objective of the CHIME study was to assess cardiorespiratory events documented by home moni- toring in infants at high epidemiologic risk for SIDS. The infants examined in this study were selected from among 228 infants delivered at Kapiolani Medical Center for Woman and Children, Honolulu, Hawaii, who participated in the CHIME study. The local institutional Review Board provided ethical approval for the study. Cry samples were collected from 23 HT and 6 siblings of SIDS infants. The HT infants were randomly selected from the entire group of HT participants. The SIDS siblings comprised the total number of such participants among the sample of 228 infants. Informed consent was obtained from each of the 29 mothers. The pregnancy and delivery of the HT infants were without complications. The mean gestational age of the HT infants was 39.5 weeks $( \pm 1.0 \mathrm{wk})$ with an average birth weight of $3312 \mathrm{~g}( \pm 326 \mathrm{~g})$. The mean Apgar scores at $1 \mathrm{~min}$ and 5 min were $7.8( \pm 1.0)$ and $8.9( \pm 0.2)$, respectively. The HT infants showed no signs of apnea or idiopathic ALTEs. In addition, there was no family history of SIDS in the last 10 years. A portion of the cry data collected for the HT infants have been reported in Robb et al. (17). The data are included in the present study to serve as a comparison to the SIDS siblings.

The mean gestational age of the SIDS siblings was 38.7 weeks $( \pm 1.8 \mathrm{wk})$ with an average birth weight of $3339 \mathrm{~g}$ $( \pm 564 \mathrm{~g})$. The mean Apgar scores at $1 \mathrm{~min}$ and $5 \mathrm{~min}$ were $7.6( \pm 1.8)$ and $8.6( \pm 1.5)$, respectively. The primary criterion for inclusion in the group of SIDS siblings was that a previous full or half sibling succumbed to SIDS (documented by autopsy). For both groups of HT and SIDS siblings, additional exclusion criteria were applied to eliminate evidence of underlying clinical pathology. Infants were excluded if they had any of the following: current pneumonia confirmed by chest X-ray, congenital heart disease, ventricularperitoneal shunt, chromosomal abnormality, midface hypoplasia or cleft palate, inborn error of metabolism, caregiver currently using illicit drugs, or inability to communicate (language barrier or no telephone; 4).

Each infant was audio recorded in the laboratory within 2 weeks following birth. Cries were elicited via administration of a pain stimulus to the sole of the infant's right foot. The pain stimulus was delivered by a $5-\mathrm{cm}$ stainless steel wire attached via a spring to a heel block. One complete crying episode was obtained from each infant in a supine position. An episode of crying commenced with the first audible cry following administration of a pain stimulus. The completion of a crying episode was noted as the last audible cry that was followed by a minimum of $15 \mathrm{sec}$ of silence. All cries were audio recorded using a condenser microphone (Realistic 33-1052) coupled to a cassette recorder (Marantz PMD360). The microphone was situated at a constant $15 \mathrm{~cm}$ from Q1 the infant's mouth. An audible tone was time-locked to administration of the pain stimulus and served as an acoustic reference for measurement of cry latency.

\section{Acoustic analysis and measurements}

The entire crying episodes were analyzed using an integrated computer hardware-software package (KAY CSL-4300). Each audio-recorded crying episode was digitized at $16 \mathrm{kHz}$ 
and displayed as an amplitude-by-time waveform. A number of acoustic measurements were selected in an attempt to identify possible differences in cry physiology between HT infants and SIDS siblings. The cry features measured allowed for characterization of the acoustic signal according to respiratory and laryngeal influences. The specific measurements were as follows:

Overall cry duration: It is defined as the onset and offset of acoustic energy across the entire crying episode.

Number of expiratory cries: It is defined as the onset and offset of visible acoustic energy that was perceived to occur on the expiratory phase of the respiratory cycle. Cry segments were bound by visible breaks (or silent periods) in the waveform.

Cry ratio: It is defined as the ratio of the overall cry duration to the number of expiratory cries produced by each infant. The ratio was assumed to provide an estimate of the proportion of time each expiratory cry consumed during the entire crying episode.

Cry latency: It is defined as the time elapsed between the administration of the pain stimulus, as indicated by an audible tone, and the onset of cry phonation. The measure is assumed to reflect the time for the central nervous system (CNS) to interpret the pain stimulus.

First spectral peak (FSP): Using a pair of vertical cursors superimposed over the waveform of the entire crying episode, the nonvoiced portions of the waveform were systematically removed, leaving a continuous display of phonatory behaviour. On the basis of this edited waveform, the long-term average spectrum (LTAS) was automatically calculated through an averaging of individual fast Fourier transform computations performed every $23 \mathrm{msec}$ across the entire crying episode. An LTAS analysis serves to average out the effects of the vocal tract on the acoustic signal, leaving a representation of the vibratory patterns of the vocal folds (18). The LTAS display provided a relative amplitudeby-frequency representation of the average energy concentrations evident throughout the frequency range of $0-$ $8000 \mathrm{~Hz}$. The frequency value associated with the first amplitude peak in the spectrum (FSP) was taken as the average $\mathrm{F}_{\mathrm{o}}$ across a crying episode. Measurement of FSP across the entire crying episode is assumed to provide a more reliable and stable estimate of $\mathrm{F}_{\mathrm{o}}$ compared to measurement of individual cry utterances.

Spectral tilt (ST): The LTAS display was also used to calculate the ratio of energy (sum of amplitudes) between 0$1000 \mathrm{~Hz}$ and $1000-5000 \mathrm{~Hz}$. The resultant value provides a neurophysiological representation of how quickly amplitudes of harmonics decline (18).

High-frequency energy (HFE): On the basis of the same LTAS display used to measure FSP and ST, the total measured energy (sum of amplitudes) between 5000 and 8000 $\mathrm{Hz}$ (35) was calculated to estimate HFE. The amount of HFE is related to the presence of noise elements during cry phonation $(14,19)$.
Table 1 Acoustic features of crying episodes obtained from healthy full term (HT) infants and siblings of SIDS infants*

\begin{tabular}{llll} 
Cry feature & HT infants & SIDS siblings & p-level \\
\hline Number of expiratory cries & $17.8(6.33)$ & $14(8.28)$ & 0.18 \\
Cry duration (sec) & $28.32(9.55)$ & $25.34(12.98)$ & 0.53 \\
Cry ratio & $1.57(0.43)$ & $1.81(0.50)$ & 0.24 \\
Cry latency (sec) & $1.10(0.33)$ & $1.63(1.50)$ & 0.11 \\
HFE & $1535(448)$ & $1834(382)$ & 0.14 \\
FSP (Hz) & $463(45)$ & $503(37)$ & 0.05 \\
ST & $0.256(.06)$ & $0.201(.01)$ & 0.03 \\
\hline
\end{tabular}

*The features include the mean number of expiratory cries, mean overall crying episode duration, mean ratio of overall cry duration-to-number of expiratory cries, mean cry latency, high-frequency energy (HFE), first spectral peak (FSP) and the spectral tilt (ST) of the crying episode. Standard deviations are shown in parentheses. Results of significance testing ( $p$ ) between the two infant groups for each cry feature are also shown.

\section{RESULTS}

The results obtained for the acoustic analysis of cries are listed in Table 1. A series of two-tailed $t$-test were performed to determine whether the groups of HT infants and SIDS siblings differed significantly for each of the cry features. Among the various cry features, two significant differences were identified between the two groups, specifically FSP and ST.

\section{DISCUSSION}

The present findings would indicate that some, but not all, features of crying differ between HT infants and SIDS siblings. The FSP of SIDS siblings was significantly higher compared to the HT group. In addition, the ST of the cries produced by SIDS siblings was significantly lower than the HT group. The high FSP obtained for the SIDS siblings is probably a result of stressful response to the pain stimuli. The present group of SIDS siblings was generally heavier than the HT infants. Therefore, the high FSP obtained for the SIDS siblings is unlikely due to physical size differences in vocal folds between the two infant groups. During the stress of pain, the laryngeal musculature is tightened and this has the effect of raising $F_{o}$ (19). The shallow ST demonstrated by the SIDS siblings indicates that the amplitude of the harmonic components comprising the cry remained elevated across the acoustic spectrum. Alternatively, cries of the HT infants were characterized by a steep ST, suggesting that the lower harmonics dominated the spectrum. Lofqvist and Mandersson (18) state that a shallow ST in combination with considerable HFE is representative of hyperadductional vocal fold behaviour. Although HFE was not significantly higher among the SIDS siblings compared to the HT group, the overall mean HFE was noticeably larger among the SIDS siblings. Considering the exploratory nature of this study and a general trend for HFE to be higher among the SIDS siblings ( $\mathrm{p}=0.14$ ), it seems likely that the combined results obtained for ST, FSP and HFE are reflective of hyperadductional vocal fold vibratory behaviour among SIDS siblings. 
Based on past research for SIDS siblings (16), it was predicted that the present group of SIDS siblings would produce cries with significantly greater energy in the upper frequency spectrum, along with the possibility of a lower $\mathrm{F}_{\mathrm{o}}$ compared to HT infants. The present HFE and ST results are in agreement with past reports of high spectral energy in SIDS siblings. However, the FSP results contrast with the previous $\mathrm{F}_{\mathrm{o}}$ findings of Colton et al. The likely explanation for this difference concerns the manner of cry sampling. Colton et al. analyzed cries that were produced spontaneously by infants compared to pain-elicited cries used in the present study. Past research has indicated that spontaneous cries are likely to be produced with a lower $\mathrm{F}_{\mathrm{o}}$ compared to pain cries (20).

Although the cries of the SIDS siblings differed significantly from those of the HT group with regard to measures of vocal fold vibratory behaviour, no such differences were found related to temporal parameters of crying. Cry duration and rate of crying are often used to estimate the role of the respiratory system in regulating cry behaviour. The average cry duration of the two groups differed by only $3 \mathrm{sec}$, and the proportion of time each expiratory cry consumed during the entire crying episode (cry ratio) was also similar. The findings obtained for these measures, paired with the significant findings for ST and FSP reinforce the suggestion that the SIDS siblings and HT infants differed primarily with regard to their laryngeal behaviour.

We hesitate to discard the present results obtained for cry latency. The SIDS siblings initiated crying following a pain stimulus approximately $500 \mathrm{msec}$ later than the HT group. In spite of a small sample of SIDS siblings, the difference in cry latency between the two groups approached significance $(p=0.11)$. The trend for the SIDS siblings to show longer cry latency than HT infants provides support for prior contentions that these infants may have a generalized delay in the brain circuitry responsible for central arousal $(7,21)$. This delayed arousal to initiate cry, paired with subsequent hyperadductory vocal fold vibratory behaviour suggests that SIDS siblings demonstrated a differential response to pain stimuli compared to HT infants.

So how do the present results compare with past research identifying acoustic features of crying in SIDS infants? Assuming SIDS infants and their siblings are more alike than different, there is an expectation that similar crying behaviours would be apparent. Based on the acoustic data reported in past SIDS studies (13-15), the present data provide a hint of a SIDS crying profile. One feature that appears to clearly differentiate SIDS infants/siblings from HT infants is in the $\mathrm{F}_{\mathrm{o}}$ (or FSP) of crying. Both Stark and Nathanson (13) and Corwin et al. (15) found a significantly high $\mathrm{F}_{\mathrm{o}}$ in their SIDS infants, and a similar result was found in the current group of SIDS siblings. In addition, a prevailing feature in cries of both SIDS infants and SIDS siblings is the occurrence of unusually pronounced energy in the highest spectral regions of the cry $(14,15)$. Although there are methodological issues related to past SIDS studies that preclude considering other possible acoustic indicators (such as cry latency and formant frequencies), the collective re- sults would suggest that the laryngeal behaviour of crying produced by SIDS infants and their siblings provide a diagnostic marker of a health condition that should not be ignored.

\section{ACKNOWLEDGEMENTS}

The CHIME study was supported by the National Institute of Child Health and Human Development grants (NICHD) HD: 29067, 29071, 28971, 29073, 29060, 29056 and 34625. Additional support was provided by the Chun Foundation for Medical Research in Pediatrics. We thank Drs Michael Corwin and Larry Tinsley for their assistance during various stages of this project.

\section{References}

1. Kattwinkel J, Hauck F, Keenan M, Malloy M, Moon R. The changing concept of sudden infant death syndrome: diagnostic coding shifts, controversies regarding the sleeping environment, and new variables to consider in reducing risk. Pediatrics 2005; 116: 1245-55.

2. Arias E, MacDorman M, Strobino D, Guyer B. Annual summary of vital statistics-2000. Pediatrics 2003; 112: 1215-30.

3. Hunt C. Sudden infant death syndrome and other causes of infant mortality. Am J Respir Crit Care Med 2001; 164: 346-57.

4. Crowell D, Brooks L, Corwin M, Davidson-Ward S, Hunt C, et al. Ontogeny of arousal. J Clin Neurophysiol 2004; 21: 1-11.

5. Steinschneider A. Prolonged apnea and the sudden infant death syndrome: clinical and laboratory observations. Pediatrics 1972; 50: 646-54.

6. Hunt C. Sudden infant death syndrome. In: Behrman R, Kliegman R, Jenson H, editors. Nelson textbook of pediatrics. 16th ed. Philadelphia, PA: Saunders Co., 2000.

7. Gislason T, Johannsson JH, Haraldsson A, Olafsdottir BR, Jonsdottir $\mathrm{H}$, et al. Familial predisposition and cosegregation analysis of adult obstructive sleep apnea and the sudden infant death syndrome. Am J Respir Crit Care Med 2002; 166: 833-8.

8. Irgens LM, Oyen N, Skjaerven R. Recurrence of sudden infant death syndrome among siblings. Acta Paediatr Suppl 1993; 82 Suppl 389: 23-5.

9. Carroll JL, Siska EL. SIDS: counselling parents to reduce the risk. Am Fam Physician 1998; April: 1566-72.

10. Barr RG, Hopkins B, Green JA. Crying as a sign, a symptom, and a signal. Cambridge: Cambridge University Press, 2000.

11. Soltis J. The signal functions of early infant crying. Behav Brain Sci 2004; 27: 443-90.

12. LaGasse L, Neal A, Lester B. Assessment of infant cry: acoustic cry analysis and parental perception. Mental Retard Dev Disabil Res Rev 2005; 11: 83-93.

13. Stark RE, Nathanson S. Unusual features of cry in an infant dying suddenly and unexpectedly. In: Bosma J, Showacre J, editors., Development of upper respiratory anatomy and function: implications for the sudden infant death syndrome. Washington, DC: US Printing Office, 1975.

14. Colton R, Steinschneider A. The cry characteristics of an infant who died of the sudden infant death syndrome. J Speech Hear Dis 1981; 46: 359-63.

15. Corwin M, Lester B, Sepkoski C, Peucker M, Kayne H, Golub H. Newborn acoustic cry characteristics of infants subsequently dying of sudden infant death syndrome. Pediatrics 1995; 96: 73-7. 
16. Colton R, Steinschneider A, Black L, Gleason J. The newborn infant cry: its potential implications for development and SIDS. In: Lester BM, Boukydis CF, editors. Infant crying: theoretical and research perspectives. New York: Plenum Press, 1985.

17. Robb MP, Crowell D, Dunn-Rankin P. Cry analysis in infants resuscitated for apnea of infancy. Int J Pediatr Otorhinolaryngol (in press).

18. Lofqvist A, Mandersson B. Long-time average spectrum of speech and voice analysis. Folia Phoniatr 1987; 39: 221-9.
19. Goberman AM, Robb MP. Acoustic examination of preterm and full-term infant cries: the long-time average spectrum. $J$ Speech Lang Hear Res 1999; 42: 850-61.

20. Lind K, Wermke K. Development of the vocal fundamental frequency of spontaneous cries during the first 3 months. Int $J$ Pediatr Otorhinolaryngol 2002; 64: 97-104.

21. Calenda PL, Mallet E, Calenda E. Siblings of sudden infant death syndrome and near miss in about 30 families: is there a genetic factor? Med Hypotheses 2000; 54: 408-11. 
Queries

Q1 Author: Please provide manufacturer's details of all the products mentioned in the article.

Q2 Author: Please provide the name of one more author in Refs. 4 and 7.

Q3 Author: Please provide volume number in Ref. 9.

Q4 Author: Please provide country/state (as applicable) in Ref. 10.

Q5 Author: Please update Ref. 17. 


\section{Please correct and return this set}

Please use the proof correction marks shown below for all alterations and corrections. If you wish to return your proof by fax you should ensure that all amendments are written clearly in dark ink and are made well within the page margins.

\begin{tabular}{|c|c|c|}
\hline Instruction to printer & Textual mark & Marginal mark \\
\hline Leave unchanged & ... under matter to remain & ( ) \\
\hline $\begin{array}{l}\text { Insert in text the matter } \\
\text { indicated in the margin }\end{array}$ & $h$ & $\begin{array}{l}\text { New matter followed by } \\
h \text { or } h \otimes 2\end{array}$ \\
\hline Delete & $\begin{array}{l}\text { I through single character, rule or underline } \\
\text { or }\end{array}$ & $\sigma$ or $\sigma / x_{2}$ \\
\hline Substitute character or & $\longmapsto$ through all characters to be deleted & $\%(x)$ \\
\hline $\begin{array}{l}\text { substitute part of one or } \\
\text { more word(s) }\end{array}$ & $\begin{array}{l}\text { I through letter or } \\
\longmapsto \text { through characters }\end{array}$ & $\begin{array}{l}\text { new character / or } \\
\text { new characters / }\end{array}$ \\
\hline Change to italics & — under matter to be changed & $\leftarrow$ \\
\hline Change to capitals & $\equiv$ under matter to be changed & $\equiv$ \\
\hline Change to small capitals & $=$ under matter to be changed & $=$ \\
\hline Change to bold type & $\approx$ under matter to be changed & $\sim$ \\
\hline Change to bold italic & $\bar{\sim}$ under matter to be changed & $\approx$ \\
\hline Change to lower case & Encircle matter to be changed & $\neq$ \\
\hline Change italic to upright type & (As above) & 4 \\
\hline Change bold to non-bold type & (As above) & nqu \\
\hline Insert 'superior' character & $\begin{array}{l}\text { I through character or } \\
K \text { where required }\end{array}$ & $\begin{array}{l}Y \text { or } Y \\
\text { under character } \\
\text { e.g. } y^{2} \text { or } \dot{X}\end{array}$ \\
\hline Insert 'inferior' character & (As above) & $\begin{array}{l}\text { over character } \\
\text { e.g. } \hat{乏}\end{array}$ \\
\hline Insert full stop & (As above) & $\odot$ \\
\hline Insert comma & (As above) & , \\
\hline Insert single quotation marks & (As above) & $\begin{array}{l}\dot{y} \text { or } \dot{x} \text { and/or } \\
\dot{y} \text { or } \dot{x}\end{array}$ \\
\hline Insert double quotation marks & (As above) & $\begin{array}{l}\dddot{y} \text { or } \ddot{x} \text { and/or } \\
\ddot{y} \text { or } \ddot{x}\end{array}$ \\
\hline Insert hyphen & (As above) & $\mapsto$ \\
\hline Start new paragraph & 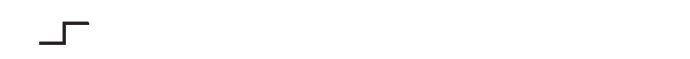 & 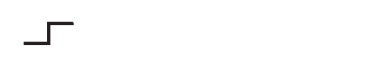 \\
\hline No new paragraph & $\curvearrowright$ & $\sim$ \\
\hline Transpose & $\sqcup$ & ص \\
\hline Close up & linking $\bigcirc$ characters & \\
\hline $\begin{array}{l}\text { Insert or substitute space } \\
\text { between characters or words }\end{array}$ & $\begin{array}{l}\text { / through character or } \\
\lambda \text { where required }\end{array}$ & \\
\hline $\begin{array}{l}\text { Reduce space between } \\
\text { characters or words }\end{array}$ & $\begin{array}{l}\text { between characters or } \\
\text { words affected }\end{array}$ & $\uparrow$ \\
\hline
\end{tabular}

